\title{
Evolution of Very Massive Stars and Type Ic Supernovae
}

Takashi Yoshida*

Department of Astronomy, Graduate School of Science, University of Tokyo

E-mail: tyoshidadastron.s.u-tokyo.ac.jp

\section{Shinpei Okita}

Department of Astronomy, Graduate School of Science, University of Tokyo E-mail: bkitadastron.s.u-tokyo.ac.jp

\section{Hideyuki Umeda}

Department of Astronomy, Graduate School of Science, University of Tokyo E-mail: umedadastron.s.u-tokyo.ac.jp

Super-luminous supernovae have been found in some recent extended supernova surveys. SN 2007bi is a Type Ic super-luminous supernova found in a metal-poor dwarf galaxy. Observational analyses indicate that this supernova has ejected $3.5-7.4 M_{\odot}$ of ${ }^{56} \mathrm{Ni}$. The large ${ }^{56} \mathrm{Ni}$ ejection can be explained by either a pair-instability $\mathrm{SN}$ of $\sim 100 M_{\odot}$ CO core or an energetic core-collapse SN of $\sim 40 M_{\odot} \mathrm{CO}$ core. In this paper, we investigate possible ranges of the main-sequence mass and the metallicity of the stars that explode as Type Ic supernovae and eject large amount of ${ }^{56} \mathrm{Ni}$. Very massive stars with a main-sequence mass $\sim 100-300 M_{\odot}$ and a metallicity $0.001<Z \lesssim 0.004$ would explode as Type Ic core-collapse supernovae producing ${ }^{56} \mathrm{Ni}$ of more than $3 M_{\odot}$. Stars with $\sim 110-150 M_{\odot}$ and $Z<0.001$ may become Type Ic supernovae if the whole H-envelope and Helayer is lost during pulsational pair-instability. More massive stars would become pair-instability supernovae with the type of supernova depending on the mitallicity. If mass loss rate is smaller than the currently adopted rate, very massive stars with $\gtrsim 170 M_{\odot}$ and $Z \sim 0.004$ would explode as Type Ic pair-instability supernovae. We also investigate the amount of ${ }^{56} \mathrm{Ni}$ ejected from Type Ic core-collapse supernovae taking into account aspherical energetic explosion of very massive stars. We adopt 43 and $61 M_{\odot}$ WO star progenitors evolved from 110 and $250 M_{\odot}$ stars with $Z=0.004$. Our results show that aspherical explosion of $110 M_{\odot}$ supernova with the opening angle $\gtrsim 45^{\circ}$ as well as $250 M_{\odot}$ supernova eject more than $3.5 M_{\odot}$ of ${ }^{56} \mathrm{Ni}$, explaining the ${ }^{56} \mathrm{Ni}$ yield observed in SN 2007bi. This suggests that aspherical core-collapse supernova explosion evolved from a very massive star is a possible explosion mechanism for SN 2007bi.

XII International Symposium on Nuclei in the Cosmos,

August 5-12, 2012

Cairns, Australia

${ }^{*}$ Speaker. 


\section{Introduction}

Recent extended supernova (hereafter abbreviated as $\mathrm{SN}$ ) surveys have raised the number of $\mathrm{SN}$ detections. Some SNe indicated new characteristics in their luminosity, light curves, spectra, etc. Super-luminous SNe are more than ten times brighter than most of Type Ia SNe [四]. These $\mathrm{SNe}$ correspond to Type IIn or Ic SNe and have a variety of light curve declines. The light curves of some of these $\mathrm{SNe}$ well reproduce the radioactive decays of ${ }^{56} \mathrm{Ni}$ and ${ }^{56} \mathrm{Co}$ while others indicate faster decline. Several origins for such SNe have been discussed; interaction with surrounding circumstellar materials, spinning-down nascent magnetars, energetic core-collapse $\mathrm{SNe}$ or pairinstability $\mathrm{SNe}$ evolved from very massive stars [四].

SN 2007bi has been observed with a maximum magnitude of -21.3 in the R-band and was recognized as a Type Ic super-luminous SN [0]. The decline of its light curve is well fitted by the radioactive decays of ${ }^{56} \mathrm{Ni}$ and ${ }^{56} \mathrm{Co}$. This $\mathrm{SN}$ was found in a metal-poor dwarf galaxy with a metallicity of $Z=(0.2-0.4) Z_{\odot}[$ [ $]$ ]. From the light curve and the spectrum analyses, the ejected ${ }^{56} \mathrm{Ni}$ amount was estimated to be $M\left({ }^{56} \mathrm{Ni}\right)=3.5-7.4 M_{\odot}$ [[]]. This amount is much larger than what is ejected from "hypernovae" such as SN 1998bw, SN 2003dh, SN 20031w (e.g., [䧃]). The ejected ${ }^{56} \mathrm{Ni}$ amount in SN 2007bi is similar to that in SN 1999as [䧃]. In order to reproduce such a huge amount of ${ }^{56} \mathrm{Ni}$, pair-instability $\mathrm{SN}$ for which the progenitor is a $\mathrm{CO}$ core with a mass of $M_{\mathrm{CO}} \sim 95-105 M_{\odot}$ was proposed as an explosion mechanism [2]. On the other hand, corecollapse SN with an explosion energy of $E=3.6 \times 10^{52} \mathrm{erg}$ from a CO-core progenitor with a mass $M_{\mathrm{CO}} \sim 43 M_{\odot}$ is also consistent with SN 2007bi in terms of the amount of ${ }^{56} \mathrm{Ni}$ [ [5]. Although there are two possible explosion mechanisms for SN 2007bi, the available mass range at the MS stage is largely different. Thus, it is important to investigate the possible ranges of mass and metallicity of massive stars which will explode as core-collapse SNe or pair-instability SNe.

Hypernova explosion is an energetic core-collapse SN explosion producing much more ${ }^{56} \mathrm{Ni}$ than "normal" SNe. Hypernova explosions of population III stars well reproduce the abundance pattern observed in extremely metal-poor stars taking into account mixing fall-back model [ 6 , 团]. Moreover, aspherical hypernova explosions well reproduce the characteristics of the mixing fallback model $[\mathbb{[}]$, thus hypernova explosion is expected to be aspherical. Observations of oxygen emission-line profiles also suggested aspherical explosions of Type Ic SNe [Q]. Therefore, the explosion of a super-luminous SN such as SN 2007bi could be an aspherical hypernova explosion.

In this study, we investigate the ranges of the mass and the metallicity of stars which explode to have core-collapse $\mathrm{SNe}$ with large ${ }^{56} \mathrm{Ni}$ production or pair-instabilitiy SNe. We calculate the stellar evolution of massive stars to the C-burning in the main-sequence mass range (main-sequence is abbreviated as MS) $M_{\mathrm{MS}}=13-300 M_{\odot}$ and the metallicity range $Z=10^{-4}-0.02$. We also investigate the ${ }^{56} \mathrm{Ni}$ yield ejected from aspherical Type Ic SN explosions evolved from very massive stars. Finally, we investigate the dependence of the ${ }^{56} \mathrm{Ni}$ yield on the asphericity of the explosion and discuss the possibility of aspherical core-collapse explosion of SN 2007bi.

\section{Massive star evolution}

We calculated the evolution of massive stars with a MS mass of $M_{\mathrm{MS}}=13-300 M_{\odot}$ and a metallicity of $Z=10^{-4}, 0.001,0.004,0.01$, and 0.02 from hydrogen burning to C-burning. Details 

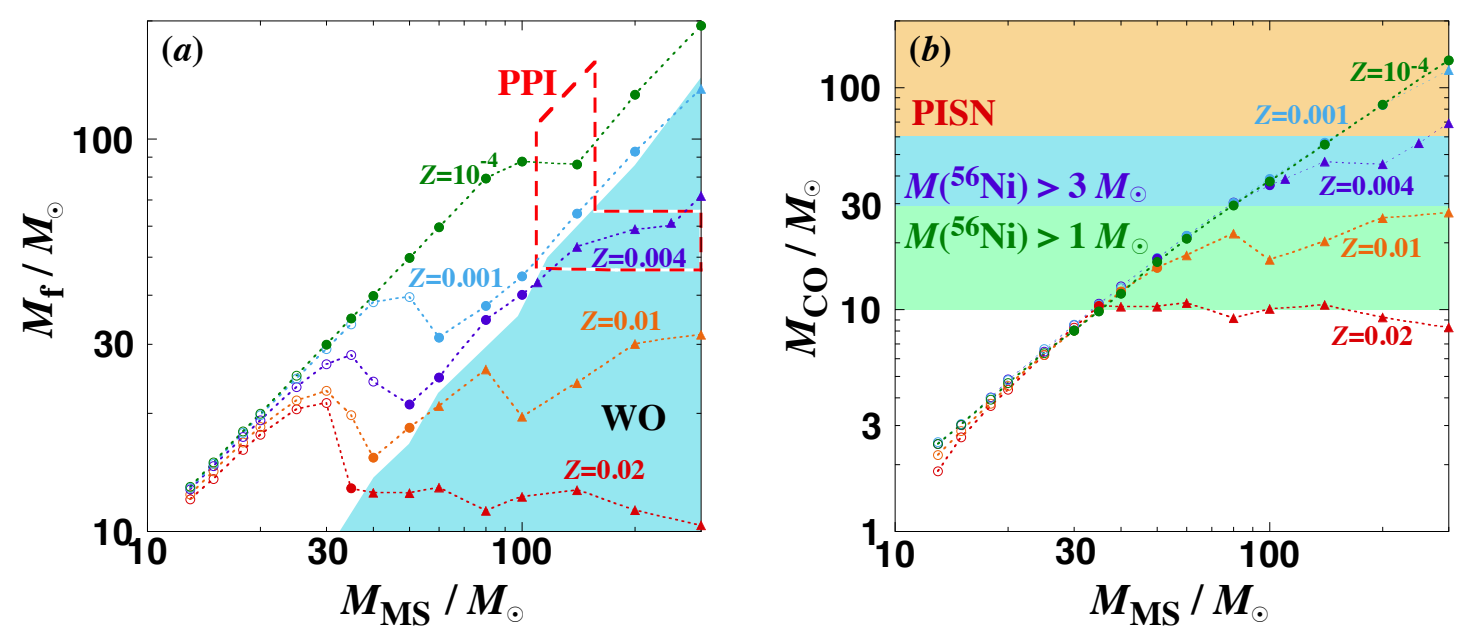

Figure 1: Final mass $(a)$ and CO-core mass $(b)$ of massive stars as a function of the MS mass. The metallicities $Z$ are 0.02 (red line), 0.01 (orange line), 0.004 (blue line), 0.001 (sky-blue line) and $10^{-4}$ (green line). Open circles, closed circles, and triangles indicate red giant stars, WN stars and WO stars, respectively. In panel $(a)$, the stars in the blue region evolve to WO stars. The stars in the region enclosed by the red-dashed line will become PPI and a part or whole of outer envelope will be lost. In panel $(b)$, the stars in the green and blue regions are expected to explode as core-collapse SNe ejecting ${ }^{56} \mathrm{Ni}$ of more than $1 M_{\odot}$ and $3 M_{\odot}$. The stars in the orange region are expected to explode as pair-instability SNe.

of the stellar evolution code are described in [एس, प]]. Mass loss effect is important for evaluating the final mass and the CO-core mass of massive stars. We adopted the following mass loss rates in massive star evolution. We used the mass loss rate during main-sequence stage as in [ए2]. We took the mass loss rate of Wolf-Rayet stars from [[1]]. The metallicity dependence was taken from [[4]]. The mass loss rate during red-giant stars was adopted from [ㄷ]]. We multiplied the metallicity dependence factor $\left(Z / Z_{\odot}\right)^{0.64}$ to the mass loss rate in this stage. This factor corresponds to the metallicity dependence of low temperature region of the mass loss rate in [ए]]. For $Z=0.004$ stars, we extended the mass range to $500 M_{\odot}$ and calculated the evolution taking into account three cases of mass loss rate (see $\$ 2.2$ for details) among $M_{\mathrm{MS}}=100-500 M_{\odot}$ stars to investigate the influence of the CO-core mass on the mass loss rate [ए]]].

\subsection{Fimal mass and CO-core mass}

Figure 1 shows the relation of the final mass $M_{\mathrm{f}}$ and CO-core mass $M_{\mathrm{CO}}$ to the MS mass. The final mass of massive stars strongly depends on the MS mass and the metallicity. At $Z=0.02$, stars with $M_{\mathrm{MS}} \gtrsim 40 M_{\odot}$ evolve to $\sim 10 M_{\odot}$ WO stars. More metal-poor stars have larger final mass and the final mass roughly increases with the MS mass. Very massive stars $\left(M_{\mathrm{MS}} \gtrsim 100 M_{\odot}\right)$ with $Z \gtrsim 0.001$ evolve to WO stars. On the other hand, very massive stars with $Z<0.001$ have $\mathrm{H}$ or $\mathrm{He}$ layer even during the C-burning. If eruptive mass loss does not occur in luminous blue variablelike events or by pulsational pair-instability (abbreviated as PPI) after the C-burning, the mass loss effect is negligible. We will discuss mass loss effect by PPI later.

The CO-core mass monotonically increases with the MS mass and shows little dependence on the metallicity for stars with $M_{\mathrm{MS}} \lesssim 35 M_{\odot}$. These stars evolve to red-giants or WN stars. When the MS mass is larger than $40 M_{\odot}$, the CO-core mass also depends on the metallicity. Stars of higher 


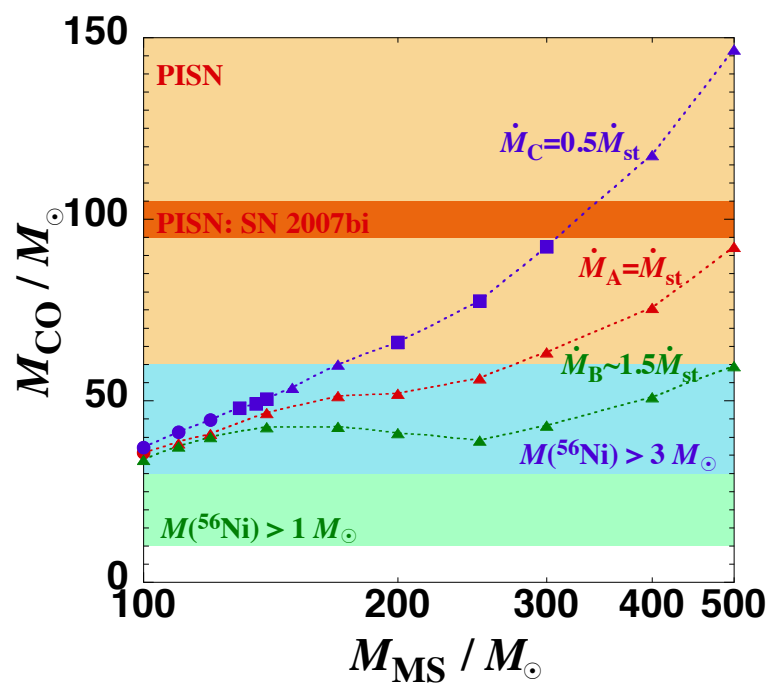

Figure 2: CO-core mass of very massive stars with $Z=0.004$ as a function of the MS mass. Red, green, and blue lines correspond to the cases (A), (B), and (C) of mass loss rate. Circles, squares, and triangles indicate WN stars, WC stars, and WO stars, respectively. Stars in green and blue regions are expected to explode as core-collapse $\mathrm{SNe}$ ejecting ${ }^{56} \mathrm{Ni}$ of more than $1 M_{\odot}$ and $3 M_{\odot}$, respectively. The stars in the light orange region will explode as pair-instability $\mathrm{SNe}$. The dark orange region indicate the range of the $\mathrm{CO}$-core mass corresponding to the progenitor of pair-instability SN explosion for SN 2007bi.

initial metallicity enhance the mass loss rate, so that metal-poorer stars indicate larger CO-core for a given MS mass. We do not see large difference between $Z=0.001$ and $10^{-4}$ stars for a given MS mass in $M_{\mathrm{MS}} \lesssim 100 M_{\odot}$ stars because of the small mass loss rate.

We discuss the possible ${ }^{56} \mathrm{Ni}$ yield for core-collapse $\mathrm{SN}$ with the relation of the CO-core mass of a massive star. Umeda \& Nomoto [16] investigated the possible ${ }^{56} \mathrm{Ni}$ yield of core-collapse SNe. They showed the relation between the ${ }^{56} \mathrm{Ni}$ yield and the CO-core mass of SN progenitors with various explosion energies. From their results, we roughly estimated the relation of the ${ }^{56} \mathrm{Ni}$ yield to the CO-core mass. We estimated that a ${ }^{56} \mathrm{Ni}$ yield of more than $1 M_{\odot}$ can be obtained from $\mathrm{SNe}$ with CO-core $M_{\mathrm{CO}} \gtrsim 10 M_{\odot}$ and an explosion energy of $1 \times 10^{52} \mathrm{erg}$. The yield of ${ }^{56} \mathrm{Ni}$ more than $3 M_{\odot}$ is obtained from a SNe with $\mathrm{CO}$ core $M_{\mathrm{CO}} \gtrsim 30 M_{\odot}$ and an explosion energy of $2 \times 10^{52} \mathrm{erg}$. On the other hand, progenitors for pair-instability $\mathrm{SNe}$ have been investigated in [ [6, [0]]. It was shown that a $\mathrm{CO}$ core larger than $60 M_{\odot}$ explodes as a pair-instability SN.

We see in Fig. $1(b)$ that very massive stars with $Z \gtrsim 0.01$ can eject ${ }^{56} \mathrm{Ni}$ of more than $1 M_{\odot}$ through core-collapse explosion. The SNe evolved from very massive stars with $0.001<Z \lesssim 0.01$ would lead to a ${ }^{56} \mathrm{Ni}$ yield of more than $3 M_{\odot}$. Thus, very massive stars with a metallicity similar to Small Magellanic Cloud have a possibility to eject ${ }^{56} \mathrm{Ni}$ of more than $3 M_{\odot}$. Very massive stars with $M_{\mathrm{MS}} \gtrsim 150 M_{\odot}$ and $Z<0.001$ can explode as pair-instability SNe. Stars with $150 \gtrsim M_{\mathrm{MS}} \gtrsim 250 M_{\odot}$ and $Z \sim 0.001$ will explode as Type II SNe. Metal poorer very massive stars will also explode as Type II SNe if eruptive mass loss does not occur after the C-burning. The stars in the region enclosed by the red line in Fig. 1(a) has a CO core of $M_{\mathrm{CO}} \sim 40-60 M_{\odot}$, thus, they will become PPI SNe and lose their outer envelope [188, [1]]. Their SN types are quite uncertain because the lost mass during PPI has not been evaluated in details yet. Stars with $M_{\mathrm{MS}} \sim 110-150 M_{\odot}$ and 
$Z \lesssim 0.001$ have an H-envelope and become PPI SNe. If the whole H-envelope and He-layer is lost by PPI, these stars will become Type Ic SNe. The radiation induced by the collision of the ejected outer envelope with the earlier ejecta during PPI is also a candidate for super-luminous $\mathrm{SNe}[\mathbb{1 8}, \mathbb{1 0}]$.

\subsection{Uncertainties in mass loss rate}

Here, we investigate the influence of the CO-core mass to uncertainties in the mass loss rate. We calculated the evolution of stars with $M_{\mathrm{MS}}=100-500 M_{\odot}$ and $Z=0.004$, corresponding to the metallicity of the host galaxy of SN 2007bi with three cases of mass loss rate as in [प0]]. Case A corresponds to the "standard" mass loss rate in this study. In case B the mass loss rate in WolfRayet phase is increased by about a factor of 1.5 compared with case A. The mass loss rate in case $\mathrm{C}$ is reduced by a factor of two from that in case A.

Figure 2 shows the relation between the CO-core mass and the MS mass in three cases of the mass loss rate. In case A, the mass range of the MS mass producing ${ }^{56} \mathrm{Ni}$ of more than $3 M_{\odot}$ from core-collapse explosion is $110 \lesssim M_{\mathrm{MS}} \lesssim 270 M_{\odot}$. Very massive stars in case B can produce ${ }^{56} \mathrm{Ni}$ of more than $3 M_{\odot}$ through core-collapse $\mathrm{SNe}$ and will not explode as pair-instability SNe. In case C, stars with $M_{\mathrm{MS}} \lesssim 170 M_{\odot}$ are expected to become core-collapse SNe. The lowest mass of WO stars is $135 M_{\odot}$. The mass range of the stars that explode as core-collapse SNe corresponding to SN $2007 \mathrm{bi}$ is $135 \lesssim M_{\mathrm{MS}} \lesssim 170 M_{\odot}$. On the other hand, pair-instability SNe similar to SN 2007bi are available in the MS mass range of $M_{\mathrm{MS}}=310-350 M_{\odot}$. Core-collapse explosion seems to be more preferable to pair-instability SN to explain SN 2007bi unless the mass loss rate is small.

\section{Aspherical core-collapse $\mathrm{SN}$ explosions of very massive stars}

In the previous section, we showed that stars with $M_{\mathrm{MS}} \sim 110-270 M_{\odot}$ will explode as corecollapse $\mathrm{SNe}$ and eject more than $3 M_{\odot}$ of ${ }^{56} \mathrm{Ni}$ with the standard mass loss. They would explode as aspherical $\mathrm{SNe}$ and the ${ }^{56} \mathrm{Ni}$ yield will depend on the asphericity of the $\mathrm{SN}$. We investigate the dependence of the ${ }^{56} \mathrm{Ni}$ yield of core-collapse $\mathrm{SNe}$ on the asphericity of the $\mathrm{SN}$ explosion.

In order to calculate aspherical core-collapse SN explosions, we calculated the evolution of stars with a MS mass of $M_{\mathrm{MS}}=110$ and $250 M_{\odot}$ and a metallicity of $Z=0.004$ to the onset of the core-collapse. The final mass and the CO-core mass of the $M_{\mathrm{MS}}=110$ and $250 M_{\odot}$ stars are $\left(M_{\mathrm{f}}, M_{\mathrm{CO}}\right)=\left(43.1 M_{\odot}, 38.8 M_{\odot}\right)$ and $\left(61.1 M_{\odot}, 56.2 M_{\odot}\right)$, respectively. Both of the stars evolved to WO stars and they are expected to explode as Type Ic SNe. The $250 M_{\odot}$ star experienced PPI during the Si-burning stage. In this study, we do not take into account eruptive mass loss during PPI. Recent study on the mass ejection from metal-poor rotating very massive stars showed that an outer layer of a few solar-masses is lost from a CO core during PPI [ए耳].

Aspherical SN explosions were calculated using two-dimensional Eulerian hydrodynamic code used in [201, [2]]. As an initial condition, the kinetic energy is injected within an opening angle $\theta_{\mathrm{op}}$ at the location of the mass cut $M_{\text {cut }}=2.0 M_{\odot}$. The range of the opening angle was chosen from $7^{\circ}$ to $90^{\circ}$. The explosion energies of the 110 and $250 M_{\odot}$ star SNe were set to be $E=5 \times 10^{52} \mathrm{erg}$ and $7 \times 10^{52} \mathrm{erg}$, respectively. An explosion with such energies roughly reproduces the photometric velocity of SN $2007 \mathrm{bi}\left(\sim 12,000 \mathrm{kms}^{-1}\right.$ ) (see Ref. [2] for details). After the hydrodynamical calculations, we calculated the explosive nucleosynthesis by postprocessing using particle trace 


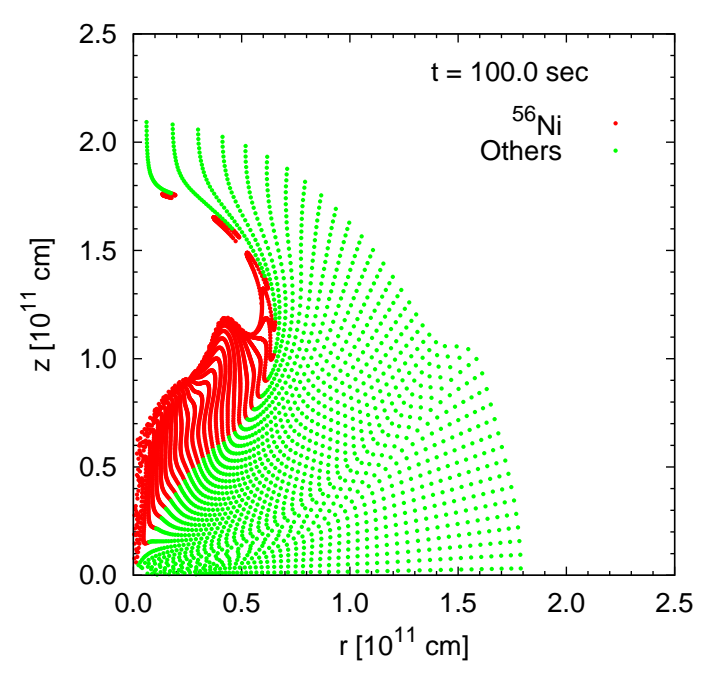

Figure 3: The special distribution of elements in the SN ejecta at $t=100 \mathrm{~s}$. The progenitor is evolved from a $250 M_{\odot}$ star. The opening angle is $\theta_{\mathrm{op}}=22.5^{\circ}$. Red and green particles indicate ${ }^{56} \mathrm{Ni}$ and O+Si dominated regions, respectively.

method. We took into account the time evolution of 5,200 Lagrangian particles during the aspherical explosions (c.f. [22] ).

Figure 3 shows the spatial distribution of elements in the SN ejecta of the $250 M_{\odot} \mathrm{SN}$ model at $t=100 \mathrm{~s}$. The kinetic energy is initially set with an opening angle $\theta_{\mathrm{op}}=22.5^{\circ}$. At the polar direction, the shock wave passes through the stellar surface and materials are blown off. Most of ${ }^{56} \mathrm{Ni}$ is produced in the polar region through explosive $\mathrm{Si}$ burning and is ejected toward the polar direction. On the equatorial plane, some materials fall into the central remnant. Since the explosive nucleosynthesis does not proceed very much, the main composition is oxygen. Finally, a mass of $\sim 10 M_{\odot}$ falls into the central region and a central remnant of about $12 M_{\odot}$ is formed.

The yields of ${ }^{56} \mathrm{Ni}$ and $\mathrm{Si}$ and the total amount of ejecta depend on the asphericity of the explosion. Figure 4 shows the yields of ${ }^{56} \mathrm{Ni}$ and $\mathrm{Si}$ and the total amount of ejecta as a function of the opening angle. In the $110 M_{\odot}$ model, the ${ }^{56} \mathrm{Ni}$ yield increases from $2.4 M_{\odot}\left(\theta_{\text {op }}=7^{\circ}\right)$ to 5.9 $M_{\odot}$ (for spherical explosion) with an opening angle. The Si yield increases from $1.1 M_{\odot}$ to $3.3 M_{\odot}$. The total amount of the ejecta changes between $25 M_{\odot}$ and $41 M_{\odot}$.

Yields of ${ }^{56} \mathrm{Ni}$ and $\mathrm{Si}$ and the total amount of ejecta in the $250 M_{\odot}$ SN model show similar dependence on the opening angle as in the $110 M_{\odot}$ SN model. The ${ }^{56} \mathrm{Ni}$ yield has a range of $3.5-$ $7.0 M_{\odot}$ according to the explosion models. The amount of ${ }^{56} \mathrm{Ni}$ produced through the explosive $\mathrm{Si}$ burning scarcely depends on the opening angle. However, about half of the produced ${ }^{56} \mathrm{Ni}$ falls into the central remnant in the case of a small opening angle. The Si yield changes in the range $3.6-5.8 M_{\odot}$. Some $\mathrm{Si}$ was produced through shell O-burning during and after PPI. The total amount of ejecta in the $250 M_{\odot} \mathrm{SN}$ model is larger than that of the $110 M_{\odot} \mathrm{SN}$ model. Ejecta of more than $46 M_{\odot}$ were obtained, even when we took the smallest opening angle $\left(\theta_{\mathrm{op}}=7^{\circ}\right)$ for the explosion.

The observations of SN 2007bi deduced an ${ }^{56} \mathrm{Ni}$ yield of 3.5-7.4M⿻. This yield is reproduced by the $110 M_{\odot} \mathrm{SN}$ model with an opening angle of $\theta_{\mathrm{op}} \gtrsim 45^{\circ}$ and the $250 M_{\odot} \mathrm{SN}$ model. Therefore, aspherical explosion of the WO star progenitors with $43-61 M_{\odot}$ would produce enough ${ }^{56} \mathrm{Ni}$ to 

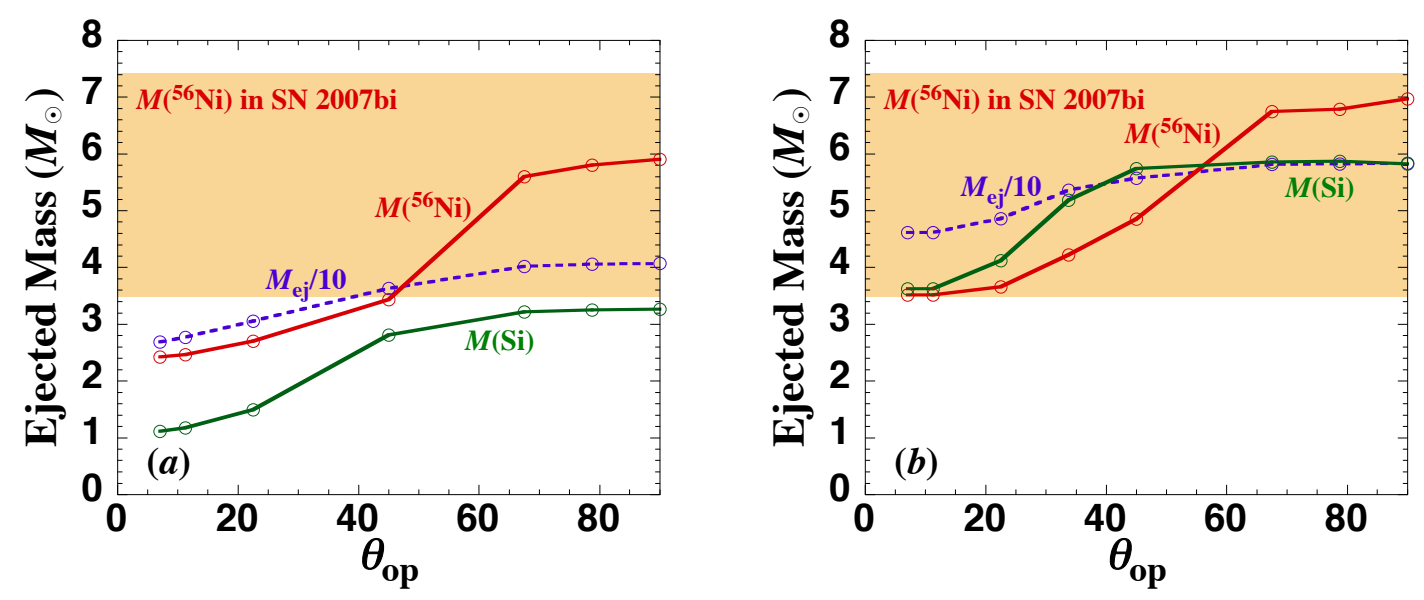

Figure 4: The yields of ${ }^{56} \mathrm{Ni}$ and $\mathrm{Si}$, and the total ejected mass of aspherical SN explosions evolved from 110 $M_{\odot}(a)$ and $250 M_{\odot}(b)$ stars as a function of the opening angle. The shaded region indicates the estimated ${ }^{56} \mathrm{Ni}$ yield based on the observations of SN 2007bi.

explain the ${ }^{56} \mathrm{Ni}$ yield observed in SN 2007bi. A SN progenitor of this mass range is considered to experience eruptive mass loss during PPI. Recent studies on PPI SNe indicated that He cores with $41-60 M_{\odot}$ lose their outer layer to become $38-49 M_{\odot}$ SN progenitors [18, [19]. Thus, even if outer layer of the $61 M_{\odot}$ WO star is lost, the SN progenitor will have a mass $M_{\mathrm{f}} \gtrsim 40 M_{\odot}$. We expect that the resultant aspherical explosion also produces ${ }^{56} \mathrm{Ni}$ as in the ${ }^{56} \mathrm{Ni}$ yield of SN 2007bi.

The amount of ${ }^{56} \mathrm{Ni}$ in SN 2007bi is reproduced by an aspherical core-collapse SN explosion of a $M_{\mathrm{MS}} \sim 110-270 M_{\odot}$ star and a pair-instability SN of a $95-105 M_{\odot} \mathrm{CO}$ core. The total ejected mass and the yields of the core-collapse SN are quite different from those of the pair-instability SN. The ejecta mass of the core-collapse $\mathrm{SN}$ is about $50-60 M_{\odot}$. This amount is about half of the ejecta mass of the pair-instability SN. The Si yield also has a large difference between the two SNe. The Si yield of the $250 M_{\odot}$ core-collapse $\mathrm{SN}$ is $3.6-5.8 M_{\odot}$. On the other hand, a pair-instability SN of a $100 M_{\odot}$ CO-core progenitor ejects $\mathrm{Si}$ of $\sim 23 M_{\odot}$ [미]. The observed values of the ejecta mass and the Si yield have large uncertainties. If these amounts are more precisely determined from observations, they will help making stronger constraints on the explosion mechanism of SN 2007bi.

Note that we did not take into account the effect of stellar rotation. The evolution of rotating very massive stars was investigated in [22]]. They suggested that $M_{\mathrm{MS}}=160-175 M_{\odot}$ rotating stars with the metallicity of the Small Magellanic Cloud will explode as pair-instability SNe corresponding to SN 2007bi.

\section{Conclusions}

We investigated the ranges of stellar mass and metallicity of either i) core-collapse $\mathrm{SNe}$ ejecting large amount of ${ }^{56} \mathrm{Ni}$ or ii) pair-instability SNe. When the metallicity is $0.001<Z \lesssim 0.004$, very massive stars $\left(100 \lesssim M_{\mathrm{MS}} \lesssim 300 M_{\odot}\right)$ would explode as Type Ic core-collapse SNe with large ${ }^{56} \mathrm{Ni}$ production. Stars with $M_{\mathrm{MS}} \sim 110-140 M_{\odot}$ and $Z<0.001$ may become Type Ic PPI $\mathrm{SNe}$ if the whole $\mathrm{H}$-envelope and He-layer is lost by eruptive mass loss during PPI. Metal-poorer 
$(Z \lesssim 0.001)$ very massive stars with the CO core of more than $60 M_{\odot}$ can become pair-instability $\mathrm{SNe}$ and their SN types would be Type Ib or Type II. If the mass loss rate is smaller, the stars with $M_{\mathrm{MS}}=310-350 M_{\odot}$ would become Type Ic pair-instability SNe corresponding to SN 2007bi.

We also investigated aspherical core-collapse SN explosions evolved from $M_{\mathrm{MS}}=110$ and $250 M_{\odot}$ stars with a metallicity of $Z=0.004$ to explain the ${ }^{56} \mathrm{Ni}$ yield of SN $2007 \mathrm{bi}$ by aspherical core-collapse explosion. The explosion of either a $110 M_{\odot}$ SN with $E=5 \times 10^{52} \mathrm{erg}$ and $\theta_{\mathrm{op}} \gtrsim 45^{\circ}$ or a $250 M_{\odot} \mathrm{SN}$ with $E=7 \times 10^{52} \mathrm{erg}$ and $\theta_{\mathrm{op}} \geq 7^{\circ}$ ejects enough ${ }^{56} \mathrm{Ni}$ to reproduce the obsevered ${ }^{56} \mathrm{Ni}$ yield of SN 2007bi. Thus, aspherical core-collapse SN explosion evolved from a very massive star is a possible explosion mechanism for SN 2007bi.

We thank Hamid Hamidani for reading our manuscript and giving variable comments. This work was supported by JSPS KAKENHI Grants (20041005, 20105004, 20540284).

\section{References}

[1] R. M. Quimby, et al., Nature 474 (2011) 487.

[2] A. Gal-Yam, et al., Nature 462 (2009) 624.

[3] D. R. Young, et al., A\&A 512 (2010) A70.

[4] K. Nomoto, N. Tominaga, H. Umeda, C. Kobayashi, and K. Maeda, Nucl. Phys. A777 (2006) 424.

[5] T. Moriya, N. Tominaga, M. Tanaka, K. Maeda, and K. Nomoto, ApJL 717 (2010) L83.

[6] H. Umeda and K. Nomoto, ApJ 565 (2002) 385.

[7] H. Umeda and K. Nomoto, ApJ 619 (2005) 427.

[8] N. Tominaga, ApJ 690 (2009) 526.

[9] K. Maeda, et al., Science 319 (2008) 1220.

[10] T. Yoshida and H. Umeda, MNRAS 412 (2011) L78.

[11] H. Umeda, T. Yoshida and K. Takahashi, Prog. Theor. Exp. Phys. 01 (2012) A302.

[12] J. S. Vink, A. de Koter and H. J. G. L. M. Lamers, $A \& A 369$ (2001) 574.

[13] T. Nugis and H. J. G. L. M. Lamers, A\&A 360 (2000) 227.

[14] J. S. Vink and A. de Koter, $A \& A 442$ (2005) 587.

[15] C. de Jager, H. Nieuwenhuijen and K. A. van der Hucht, ApJS 369 (1988) 574.

[16] H. Umeda and K. Nomoto, ApJ 678 (2008) 1014.

[17] A. Heger and S. E. Woosley, ApJ 567 (2002) 532.

[18] S. E. Woosley, S. Blinnikov, \& A. Heger, Nature 450 (2007) 390.

[19] E. Chatzopoulos and J. C. Wheeler, (2012) ApJ 760 (2012) 154.

[20] S. Okita and H. Umeda, submitted to ApJ.

[21] S. Okita, H. Umeda and T. Yoshida, XII International Symposium on Nuclei in the Cosmos, PoS(NICXII) (2012) 130.

[22] S. Fujimoto, K. Kotake, M. Hashimoto, M. Ono, and N. Ohnishi, ApJ 738 (2011) 61.

[23] N. Yusof, R. Hirschi and H. A. Kassim, Proceedings IAU Symposium 279 (2012) 431. 